Feature Article

\title{
Redox-active host-guest supramolecular assemblies of peptides and proteins at surfaces
}

\author{
Jenny Brinkmann, Dorothee Wasserberg, Pascal Jonkheijm*
}

Bioinspired Molecular Engineering Laboratory of the MIRA Institute for Biomedical Technology and Technical Medicine and the Molecular Nanofabrication Group of the MESA ${ }^{+}$Institute for Nanotechnology, University of Twente, P.O. Box 217, 7500 AE Enschede, Netherlands

\section{A R T I C L E I N F O}

\section{Article history:}

Received 18 January 2016

Received in revised form 17 March 2016

Accepted 21 March 2016

Available online 22 March 2016

\section{Keywords:}

Supramolecular chemistry

Monolayers

Cucurbituril

Cyclodextrin

Redox-active chemistry

\begin{abstract}
A B S T R A C T
The cell machinery is very complex, precisely regulated by cues in space and time. Fine tuning bioactive cues on surfaces has shown to be a crucial design criterion for cell interactions with materials. Stimuli-responsive surfaces of biomaterials and devices open up new opportunities to steer cellular fate and study cellular mechanisms. Supramolecular host-guest interactions are in itself dynamic in nature due to the noncovalent nature of the interactions between molecules, and in addition they allow for the incorporation of guests, controlled using external stimuli. This tunable reversibility makes them highly attractive for the exploration of cell-interactive surfaces for biological applications. In this feature article strategies to anchor bio- and redox-active peptides and proteins employing supramolecular host-guest chemistry are presented and discussed in the context of these surfaces interacting with cells.
\end{abstract}

\section{Contents}

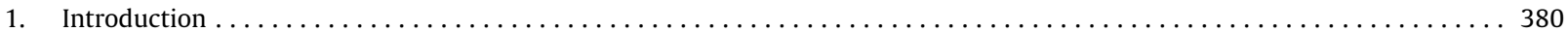

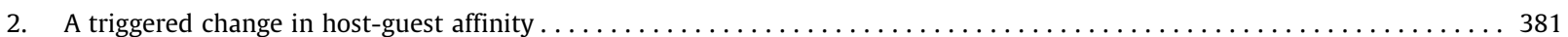

3. Anchoring of proteins conjugated with redox-active guests on host-functionalized surfaces $\ldots \ldots \ldots \ldots \ldots \ldots \ldots \ldots \ldots \ldots$

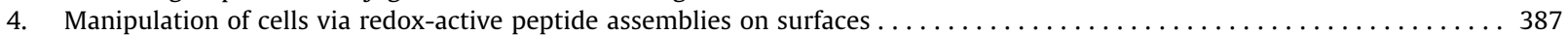

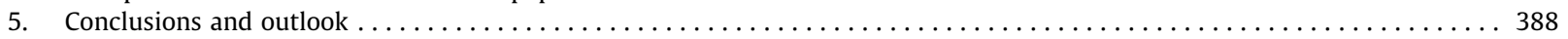

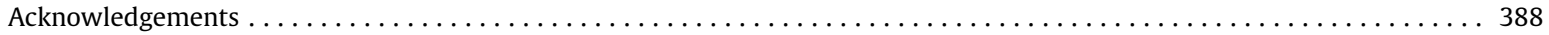

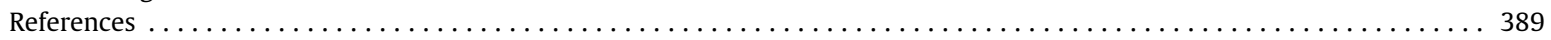

\section{Introduction}

Assemblies of peptides and proteins on arrays, beads, chips, scaffolds and aggregates play an increasingly important role in the development of bioanalytics and biomedicine [1,2]. Protein-modified surfaces are also of interest for the study of cell growth and cell differentiation [3]. The interaction of cells with modified surfaces plays an important role in tissue engineering and the design of materials for the fabrication of medical implants. In addition, the controlled immobilization of enzymes

\footnotetext{
* Corresponding author.

E-mail address: p.jonkheijm@utwente.nl (P. Jonkheijm).
} 


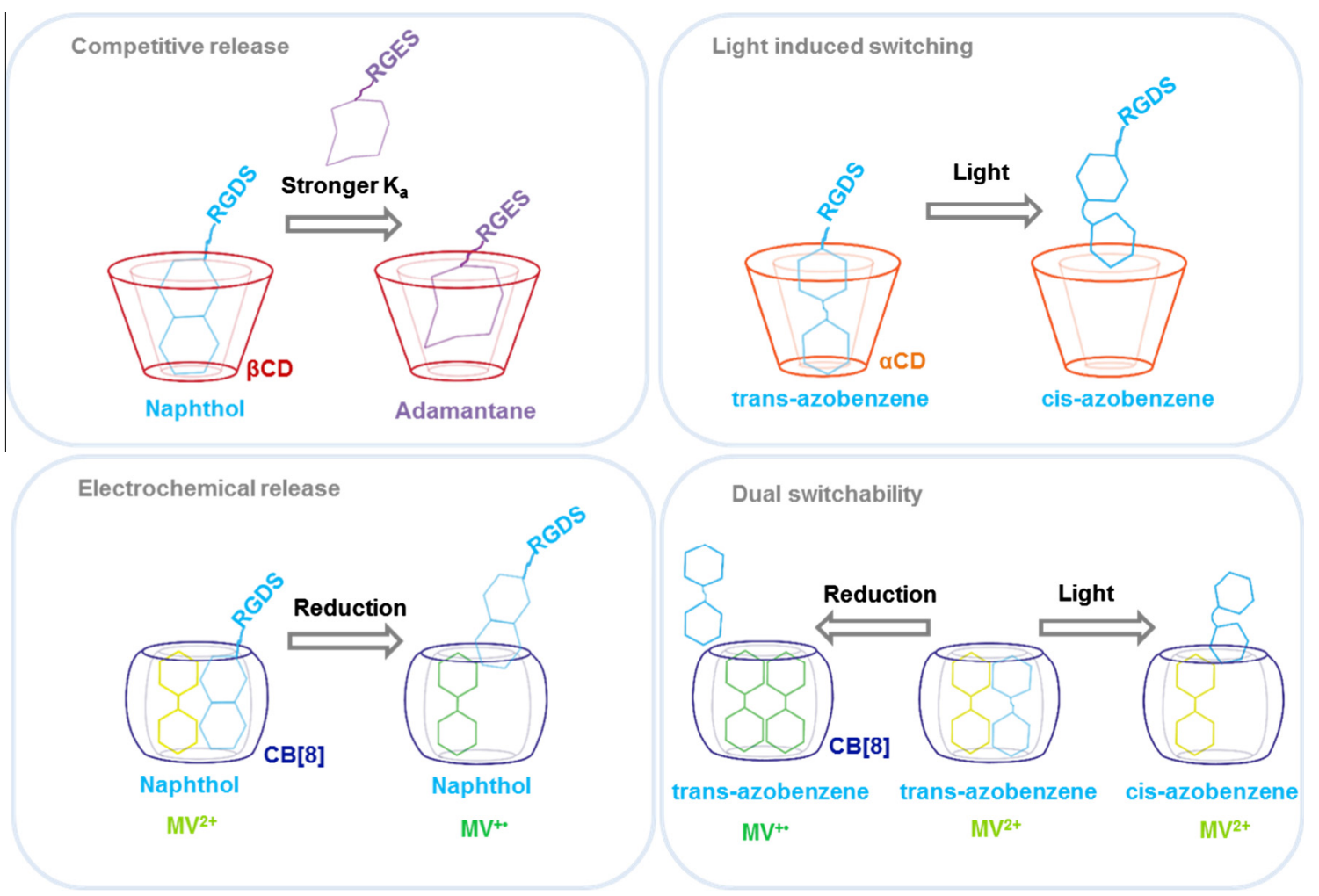

Fig. 1. Triggered change in host-guest affinity by (a) chemicals, (b) photons, (c) electrons or (d) a combination of photons and electrons. (A) Competitive release of the first guest by the second guest based on differences in binding strength to a cyclodextrin host. (B) Switching from trans to cis-azobenzene limits the interaction with the cyclodextrin host. (C) Electrochemical reduction of methylviologen leads to weakening of the charge-transfer complex with naphthol inside the $\mathrm{CB}[8]$ cavity. (D) trans-azobenzene and methylviologen can simultaneously interact with $\mathrm{CB}[8]$. Electrochemical reduction of methylviologen or light causes release of the azobenzene from the $\mathrm{CB}[8]$ cavity. Host molecules are schematically depicted.

is crucial to optimizing catalytic activities [4]. Supramolecular chemistry is a versatile tool for the assembly of peptides and proteins on surfaces, as it, in principle, allows for reversible attachment of biomolecules to the surface, not accessible via covalent immobilization techniques [5]. Additionally, supramolecular anchoring of peptides and proteins provides access to controlling the dynamics of the interaction through selecting the valency and type of interaction motifs. Furthermore, the density of biological ligands on the surface can be controlled through using photons, electrons, protons or other chemicals (Figs. 1 and 2), since often a change in e.g. a redox potential can lead to a change in affinity of the bioactive ligands with the surface.

While interfacing static, non-living systems with dynamic, living matter is an essential step in applying biomaterials in tissue regeneration, finding the optimum interface is a formidable task [1]. The highly complex composition of the extracellular matrix (ECM) and its complex interaction with the so-called molecular fingerprints on cell membranes make it a highly challenging task to create model systems. Since natural interfaces between the cell and its interacting ligands are built on non-covalent interactions, chemical systems relying on non-covalent binding such as hydrophobic, van der Waals, iondipole or hydrogen bonds are being explored and representing a promising approach to mimic natural cell-ECM interactions [1]. Self-assembly is an attractive tool and an efficient bottom-up strategy to govern thermodynamic control aiming to position ligands at predefined locations and the modulation of those positions [1]. An appealing self-assembly strategy to create interfaces to cells is the use of supramolecular host-guest chemistry based on cucurbit[n]urils (CB[n]) and cyclodextrins (CD). They are highly attractive due to their ability of binding various guest molecules in physiologically relevant milieu. Moreover, these host-guest interactions are sensitive to e.g. redox-triggered affinity changes (Figs. 1 and 2).

\section{A triggered change in host-guest affinity}

$\mathrm{CB}[n] \mathrm{s}$ are macrocyclic pumpkin-shaped molecules made of $n$ individual glycoluril monomer units. Their hydrophobic core and electronegative carbonyl rim allows for highly specific interactions with small hydrophobic guest molecules $[6,7]$. $\mathrm{CB}[7]$ recognizes hydrophobic and electroactive guests, such as ferrocene and methylviologen, with dissociation constants in the nM to pM range (Fig. 2). $\mathrm{CB}[8]$, having a larger core diameter, has the unique ability to simultaneously bind two 

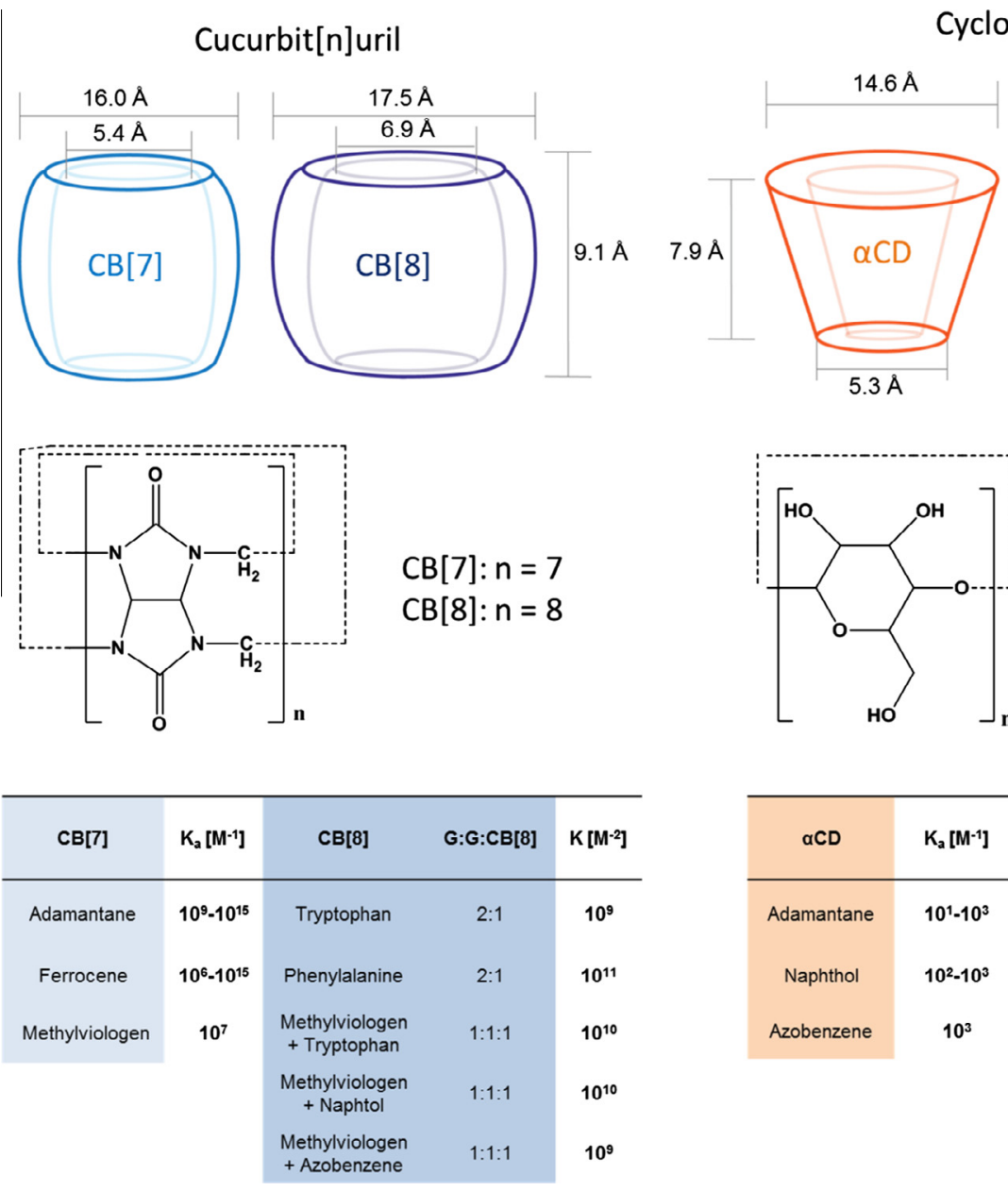

Cyclodextrin
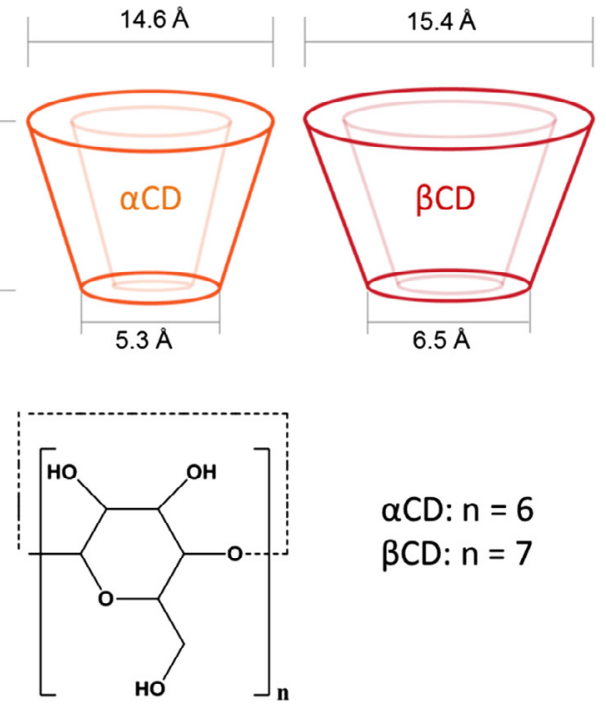

$6.5 \AA$

$$
\begin{aligned}
& \alpha C D: n=6 \\
& \beta C D: n=7
\end{aligned}
$$

\begin{tabular}{c|ccc}
\hline$\alpha C D$ & $K_{\mathrm{a}}\left[M^{-1}\right]$ & $\beta C D$ & $K_{\mathrm{a}}\left[M^{-1}\right]$ \\
\hline Adamantane & $10^{1}-10^{3}$ & Adamantane & $10^{3}-10^{5}$ \\
\hline Naphthol & $10^{2}-10^{3}$ & Naphthol & $10^{1}-10^{3}$ \\
\hline Azobenzene & $10^{3}$ & Azobenzene & $10^{3}-10^{4}$ \\
\hline & & Ferrocene & $10^{3}-10^{4}$ \\
& & Carborane & $10^{6}$ \\
\hline
\end{tabular}

Fig. 2. Chemical structures of $\alpha \mathrm{CD}, \beta C D, C B[7]$ and $\mathrm{CB}[8]$ with their molecular dimensions and examples of guest $(\mathrm{G})$ molecules with association constants $\left(K_{a}\right)$ found in solution (see e.g. Ref. [9]).

aromatic guest molecules. It can for instance incorporate an electroactive methylviologen $\left(\mathrm{MV}^{2+}\right)$ as the first guest along with naphthol, N-terminal tryptophan or azobenzene as the second guest. While in the former case oxidation of ferrocene is expected to yield a moderate change in affinity to the $\mathrm{CB}$ [7] cavity, in the latter case, reduction of methylviologen to its radical monocation weakens the ternary complex yielding a release of the second guest (Fig. 1).

CDs are nonsymmetrical cyclic oligosaccharides, interacting with hydrophobic guest molecules such as naphthol, ferrocene, adamantane and azobenzene with dissociation constants in the $\mu \mathrm{M}$ to $\mathrm{mM}$ range (Fig. 2) [8]. Exploration of these types of host-guest interactions has led to various designs of supramolecular hydrogels, polymers and nanoparticles and are reviewed elsewhere $[9,10]$. When ferrocene guests are oxidized the affinity with CD is expected to be lost.

This feature article focuses on reviewing our work dealing with bioconjugating peptides and proteins with redox-active moieties and their interaction with different types of host-functionalized surfaces (Section 3). Their use as bioactive interfaces to study electro-responsive interactions with cells will also be described (Section 4).

Another approach to change the affinity between host and guest is to conjugate a light-responsive azobenzene to the peptide or protein. Azobenzene is known to interact with supramolecular hosts (Fig. 2). Azobenzene can form an inclusion complex with for example cyclodextrin when present in the trans isomer, while in the cis-isomer a loss in affinity was found leading to a release of the guest (Fig. 1) [11]. In a sophisticated approach, both azobenzene and methylviologen can be combined to interact with $\mathrm{CB}[8]$ to create dual control (Fig. 1) [12]. As an alternative approach, release can be triggered by supply of another guest ligand with a higher association constant as compared to the initially bound guest ligand (Fig. 1).

\section{Anchoring of proteins conjugated with redox-active guests on host-functionalized surfaces}

An important example of host-guest mediated anchoring of redox-active proteins was shown by Kim and co-workers using glucose oxidase (GOx), which was randomly conjugated with ferrocene (Fc) as supramolecular guest. The Fc-Gox 


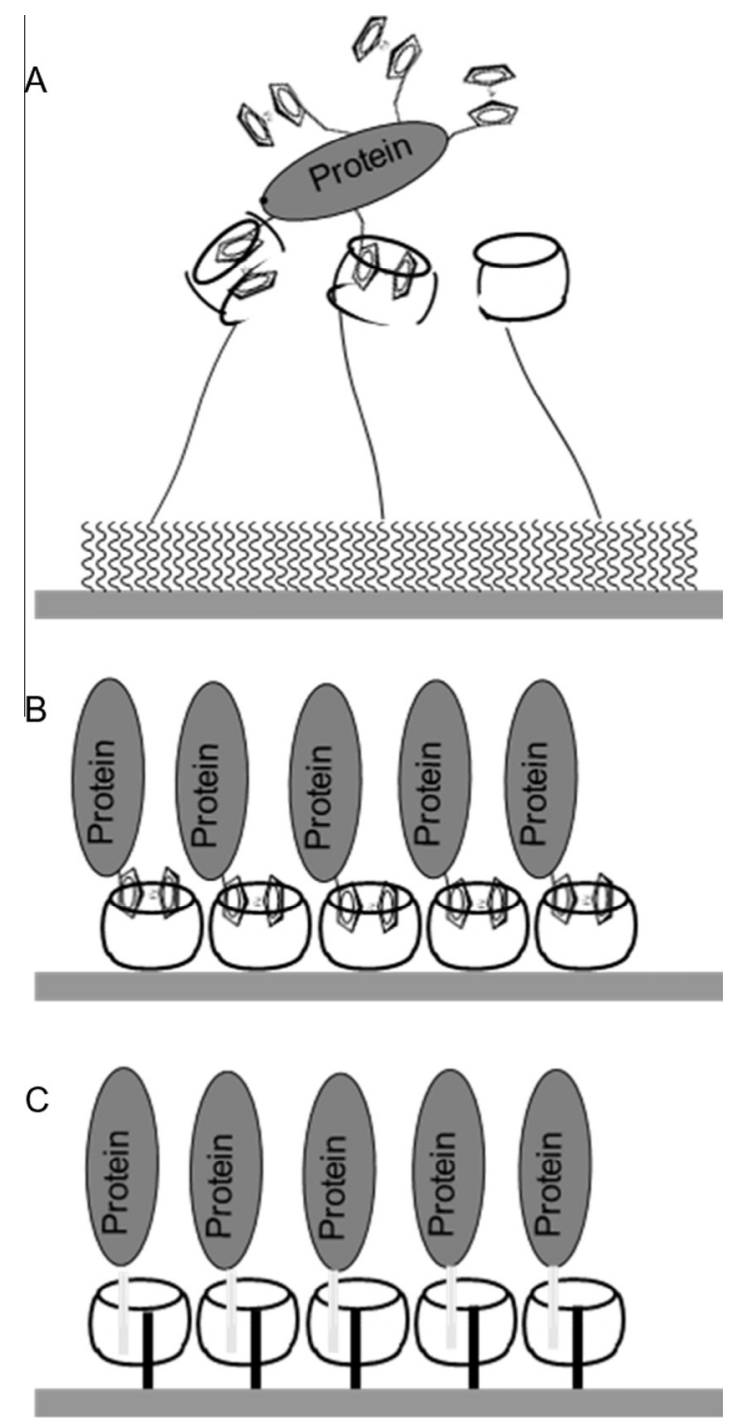

Fig. 3. Various strategies to achieve redox-active anchoring of proteins to surfaces using $\mathrm{CB}[\mathrm{n}]$ interactions.

was subsequently anchored on $\mathrm{CB}$ [7] modified surfaces, with the $\mathrm{CB}$ [7] acting as host (Fig. 3A) [13]. The proteins retained sufficient activity levels to perform glucose sensing experiments [13]. The activity of the multiple ferrocenylated enzymes was lower when compared to that of a non-labelled GOx. However, covalent anchoring of the enzyme that was based on random amide bond formation between the protein and surface showed similarly reduced activity levels [13]. In this regard, recently developed site-specific protein labelling techniques constitute an interesting alternative to random techniques [5]. Site-selective and mono-functional protein conjugation of supramolecular tags offer distinct advantages over random protein labelling and new and versatile methods to incorporate supramolecular elements are therefore urgently needed [5].

For single, site-specific labelling we employed SNAP-tag technology, which is a genetically encoded protein tag, to efficiently label proteins with guest moieties featuring an $\mathrm{O}^{6}$-benzylguanine moiety such as adamantane [14] and redoxactive ferrocene [15]. In the case of the ferrocene, ferrocene-substituted benzylguanine was covalently attached to a cysteine residue within the guanine binding pocket of the SNAP-tag (Fig. 4) [15]. For conceptual studies, we used fluorescent proteins for their robustness and intrinsic fluorescence making them ideal bio-probes for the use of fluorescence spectroscopy and microscopy. We showed that the assembly of ferrocene-functionalized SNAP-fusion proteins on cyclodextrin- and cucurbit[7]uril-coated surfaces yielded stable monolayers [15]. We also found that the binding of all ferrocene fusion proteins was specific as determined by surface plasmon resonance [15].

SNAP-tag technology adds a bulky biomolecular entity to the protein of interest and increases the sensitive nature of the entire construct. However, other site-selective ligation strategies such as the so-called expressed protein ligation only introduce a native peptide bond between the ferrocene (Fc) guest moiety and the protein of interest. In this respect, we linked a yellow fluorescent protein (YFP) bearing a C-terminal thioester to a cysteine-functionalized ferrocene via expressed protein 

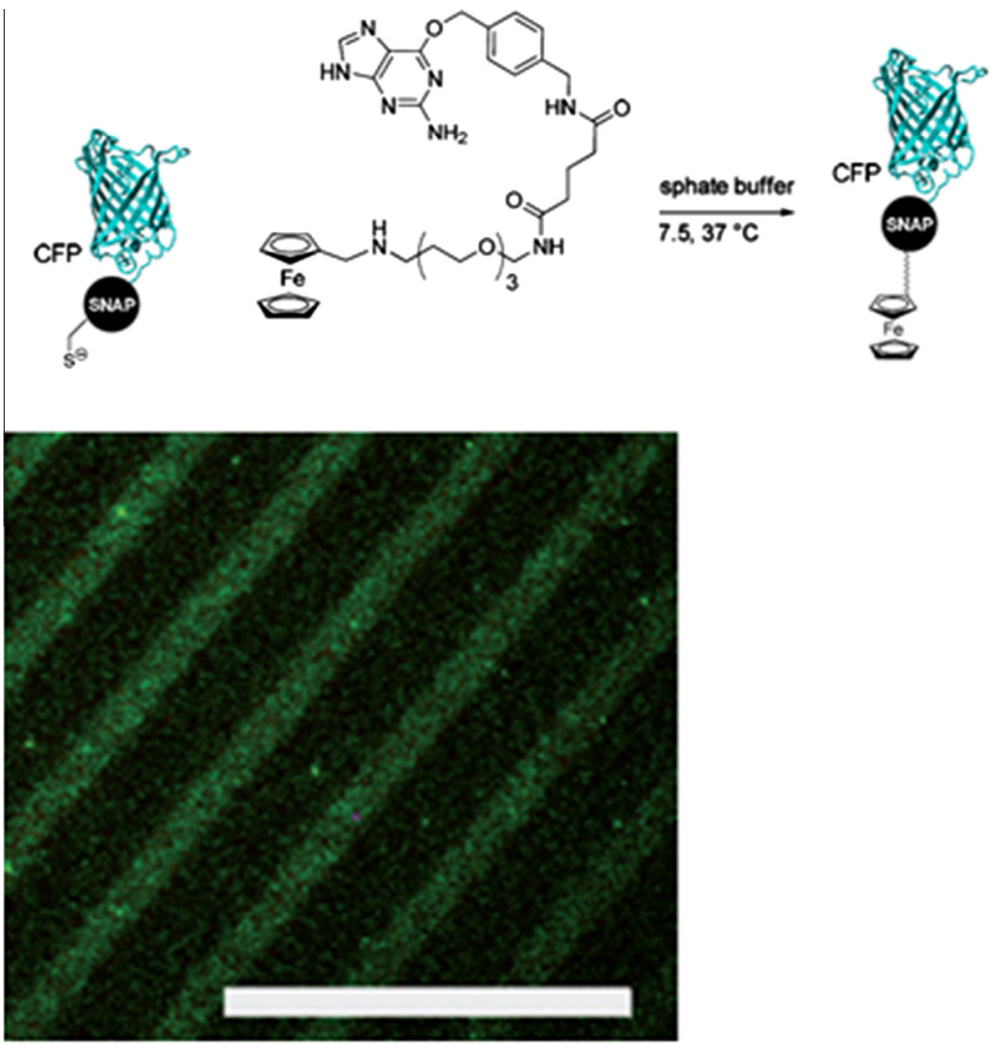

Fig. 4. Ligation of fluorescent SNAP-fusion proteins to ferrocene-benzylguanine and representative fluorescent microscopy recordings of cyclodextrin patterned surfaces (prepared via UV photolithography procedures) that were incubated with SNAP-fusion proteins bearing guest moieties. The scale bar is $50 \mu \mathrm{m}$. Adopted from Refs. [14,15].
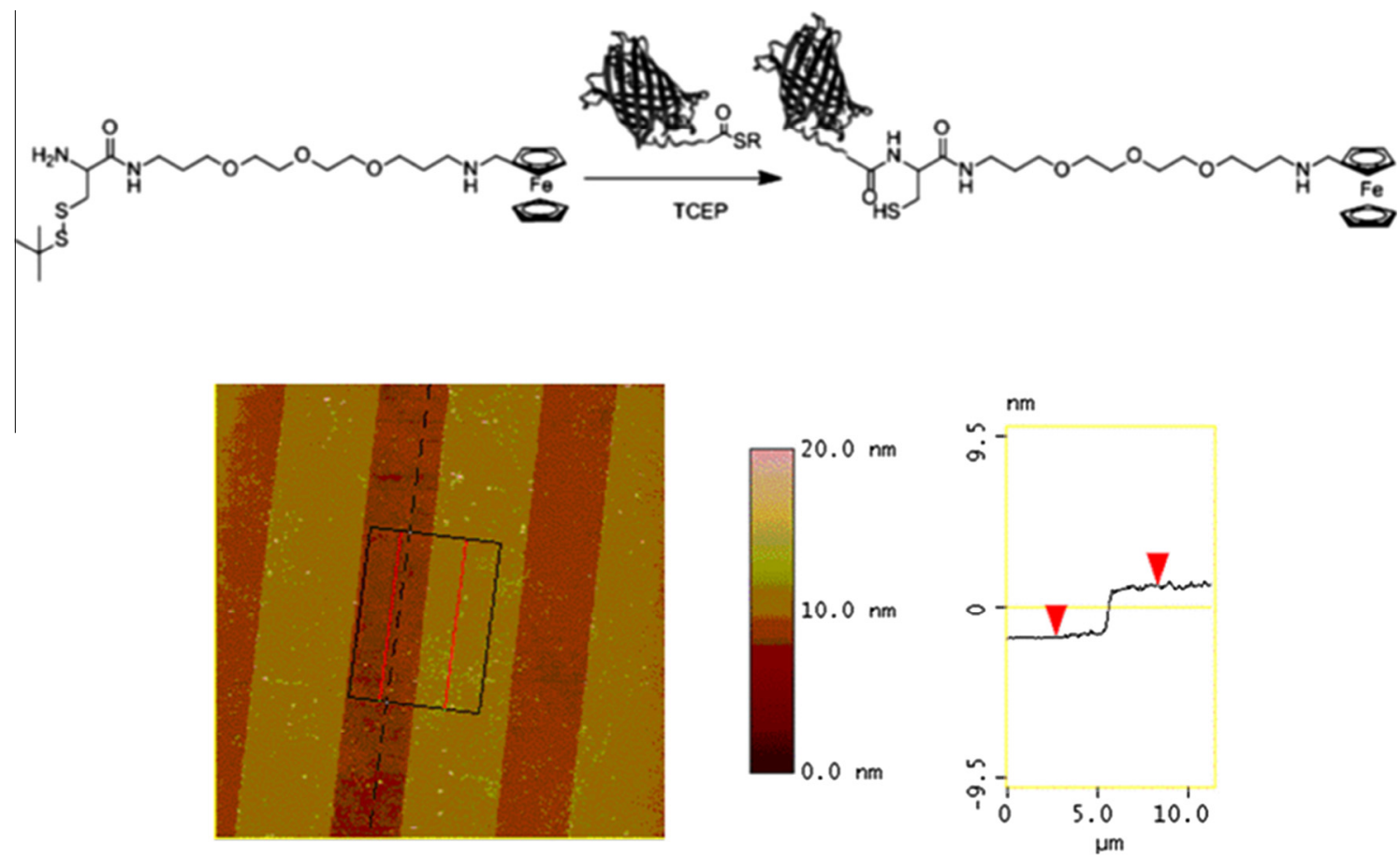

Fig. 5. Ligation of a ferrocene-cysteine derivative to YFP and subsequent anchoring of ferrocenylated YFP on a self-assembled monolayers of CB[7]. Adopted from Ref. [16]. 
ligation (Fig. 5) [16,17]. We first exploited the interaction of these ferrocenylated YFPs with self-assembled monolayers (SAMs) of CB[7] (Fig. 3B) [16]. CB[7] spontaneously adsorbs onto gold surfaces to form stable SAMs [18]. While the ferrocene complex fills the cavity of $\mathrm{CB}$ [7], the protonated amino functionality protrudes from the cavity, where it further stabilizes the interaction electrostatically by forming ion-dipole interactions with the polar carbonyl groups on CB[7]'s rim [7]. The binding constant of this host-guest system has not yet been determined on surfaces, however binding constants in comparable systems in solution are found to be in the range of $10^{11} \mathrm{M}^{-1}$ [7]. Upon incubation of a CB[7]-SAM with ferrocenylated YFP, the fluorescent proteins formed a stable and homogeneous monolayer as was shown using various analytical techniques [16]. For example, the high-resolution X-ray photoelectron (XP) spectra revealed Fe 2p binding energies of 707.9 and $720.8 \mathrm{eV}$ which are indicative of the $2 \mathrm{p}_{3 / 2}$ and $2 \mathrm{p}_{1 / 2}$ transitions of the ferrocene moiety [16]. Cyclic voltammetry (CV) measurements confirmed the presence of $\mathrm{Fe}^{\mathrm{II}}$ bound to $\mathrm{CB}[7]$ on the gold surface with scans showing reproducible one-electron waves at a scan speed of $25 \mathrm{mV} / \mathrm{s}$ [16]. Furthermore, microcontact printing, fluorescence imaging and AFM experiments all suggested that the proteins were immobilized in monolayers, that is, layers of a single protein in height and with oriented proteins through a specific monovalent $\mathrm{CB}$ [7]-ferrocene interaction [16]. The regions between the protein patterns were free of nonspecifically absorbed proteins (Fig. 5) [16]. Finally, we could show that the supramolecular protein anchoring was reversible with the use of appropriate competitive, stronger binding guest molecules [16].

In another contribution, we studied the interaction of Fc-YFP constructs with $\beta C D$ SAMs depending on the valency of the Fc-YFP constructs. A number of Fc-YFP constructs with different ferrocene valencies, (mono- and divalent) were generated by making use of the construct's intrinsic affinity for noncovalent homodimerization as well as cysteine residues for covalent homodimerization via the formation of disulfide bonds (Fig. 6) [17]. In short, one variant (Fc-dYFP) contained two point mutations that increased the affinity for noncovalent dimerization, while the so-called enhanced variant (Fc-eYFP) lacked these dimerization-promoting mutations [17]. In addition we made use of the fact that expressed chemical ligation leads to the formation of a cysteine residue (Fig. 5). The cysteine residues of the Fc-YFP constructs, are situated in such a way that they are amenable to the formation of covalent disulfide crosslinks via oxidation [17]. In a control variant (Fc-bYFP), the cysteine residues of the monovalent constructs were blocked by reaction with N-methylmaleimide [17]. The different Fc-YFP variants were assembled on SAMs of $\beta C D$ [17]. To this end, $\beta C D$ was modified with seven thioether chains to form highly ordered and densely packed SAMs on gold (Fig. 6) [17]. Clear binding events in surface plasmon resonance (SPR) sensograms were observed in the case of all Fc-YFP constructs while no binding was observed in the case ferrocene was not ligated to YFP
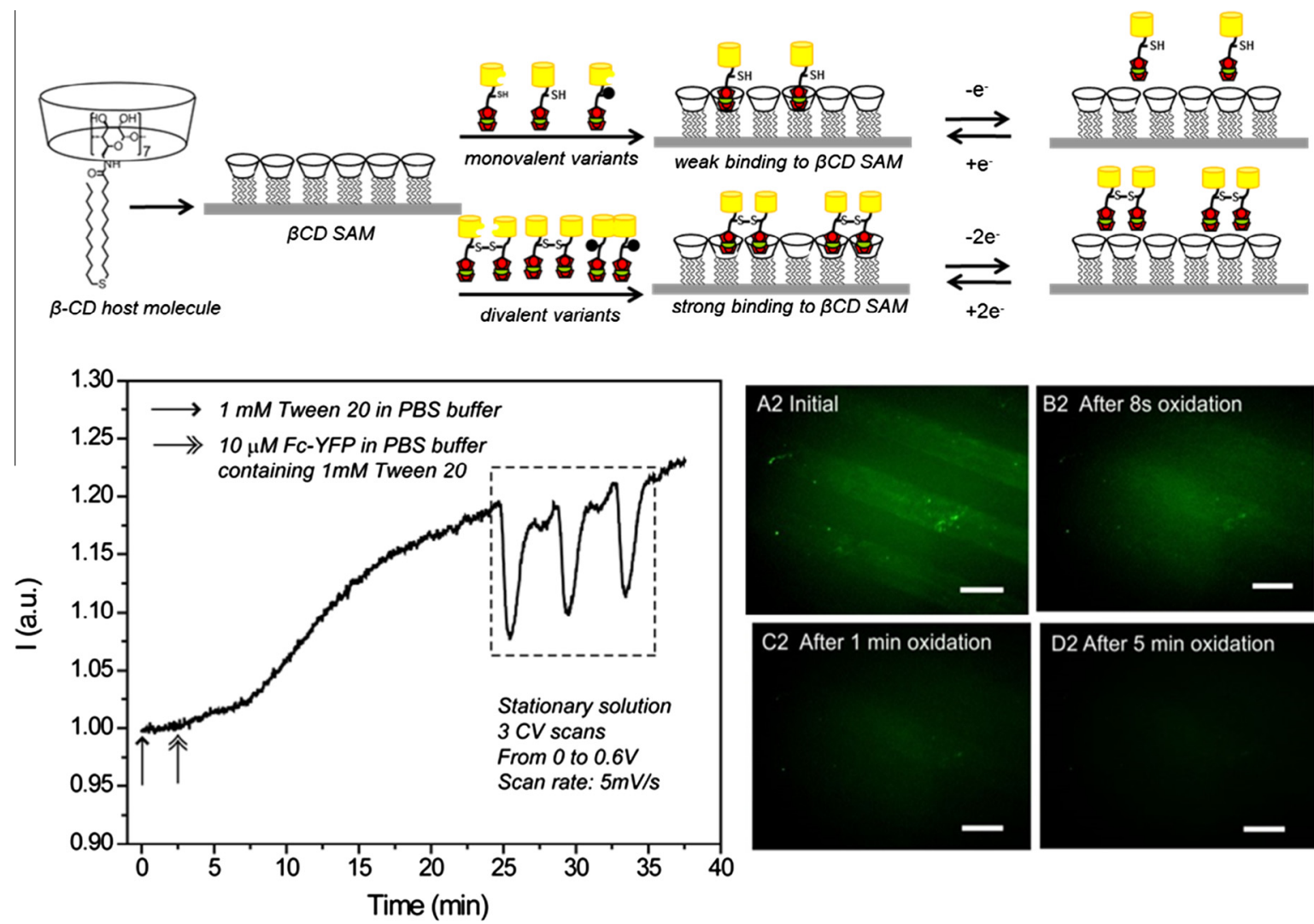

Fig. 6. SPR response while performing cyclic voltammetry and fluorescence images of patterned ferrocenylated YFP on cyclodextrin monolayers during chronoamperometric oxidation. Adopted from Ref. [17]. 
[17]. More of the covalently crosslinked dimers was adsorbed to the surface at equal concentrations when compared to their monomeric counterparts [17]. The interaction between Fc-YFPs and the surface was reversible for both the mono- and divalent variants, with the latter being bound more tightly to the host layer. Here, the weak interaction of the monovalent variants with $\beta C D$ was clearly stabilized via covalent protein dimerization of the divalent YFP constructs. However, when covalent dimerization was prevented (by blocking the cysteines), the dimerization-promoting mutations of Fc-dYFP alone could not strengthen the YFP interaction with the $\beta C D$ SAM compared to FC-eYFP monomers. The divalent construct was found to have an association constant of $2.5 \times 10^{5} \mathrm{M}^{-1}$, indicating that the proteins simultaneously interact with two surfaces-bound cyclodextrin hosts via both its ferrocence units with an intrinsic association constant of $1.2 \times 10^{3} \mathrm{M}^{-1}$ per ferrocene [17]. Uniform monolayers of Fc-YFPs, produced in patterns on $\beta C D$ surfaces, were characterized using fluorescence and atomic force microscopy measurements [17]. Fluorescence microscopy imaging and SPR measurements were carried out with in-situ performed chronoamperometry and cyclic voltammetry, respectively (Fig. 6) [17]. These studies proved the first time the feasibility of repetitive desorption and adsorption of ferrocene-tagged proteins from a supramolecular surface upon electrochemical oxidation and reduction, respectively [17]. $\beta C D$ has a weak binding affinity for ferrocene in solution ( $\mathrm{K}_{\mathrm{a}}$ on the order of $10^{3} \mathrm{M}^{-1}$ ) that is strongly diminished upon oxidation of $\mathrm{Fc}$ to $\mathrm{Fc}^{+}$. The above results describe a biomimetic system that merges the possibilities of controlling the orientation, valency, affinity, reversibility, and responsiveness. Such systems are of interest for fabricating adaptive biomimetic interfaces that are important for detailed studies of biological processes at the cellular level.

Another member of the $\mathrm{CB}[\mathrm{n}]$ family is $\mathrm{CB}[8]$, which is known to accommodate stable $1: 1: 1$ ternary complexes with an electron-deficient supramolecular guest molecule such as methyl viologen (MV) and an appropriate electron-rich guest such as alkoxynaphthalene or aromatic amino acids (Fig. 2) [6,7]. Formation of such ternary complexes is characterized by hostguest-assisted charge transfer interactions and by binding constants of $10^{11}-10^{13} \mathrm{M}^{-2}$ (Fig. 2) [6,7]. In this way CB[8] can tie together two different building blocks to form a supramolecular assembly. For example, a viologen-based guest molecule was attached by us to the surface, while a naphthol guest moiety was chemoselectively ligated to a YFP (Fig. 3C) [19]. CB [8] was used to selectively link the proteins onto MV-functionalized surfaces as evidenced by characteristic bands appearing in the recorded IR-reflective absorption and X-ray photoelectron spectra upon complex formation [19]. Two different surface anchoring routes were followed to form patterns of the such ternary complexes on the surface and evaluated by fluorescence microscopy (Fig. 7). Each anchoring route consisted of three steps: (i) attaching the viologen to the glass using reactive
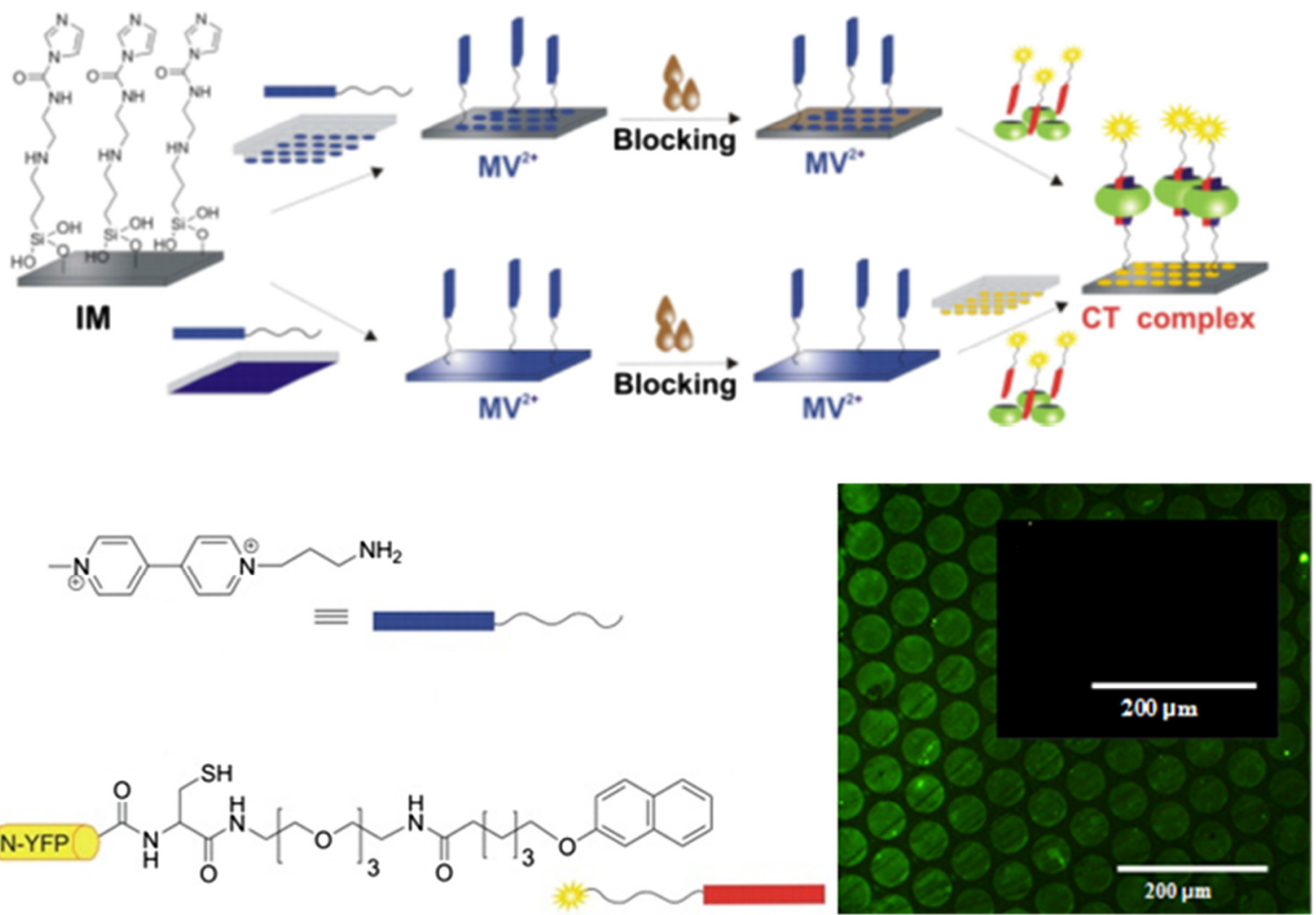

Fig. 7. Two immobilization routes for patterning ternary complexes on glass chips are depicted. Methyl viologen and naphthol-functionalized YFP are given. Fluorescence microscopy image of naphthol-YFP (inset YFP only) in ternary complex following the upper immobilization route. Adopted from Ref. [19]. 
microcontact printing on imidazolide (IM) monolayers, (ii) blocking, and (iii) either incubation (top route) or microcontact printing (bottom route) of $\mathrm{CB}$ [8] and naphthol guests (Fig. 7) [19]. In both cases uniform and stable fluorescent patterns were fabricated with a high signal-to-noise ratio [19]. Control experiments confirmed that $\mathrm{CB}[8$ ] served as a selective linking unit to form stable and homogeneous ternary surface-bound complexes as had initially been envisioned [19].

To selectively anchor proteins to surfaces in a reversible or even stimulus-responsive manner, generally requires more steps and is more complex than anchoring DNA or peptides. Mostly due to the sensitive nature of proteins, chemical treatment and/or anchoring often results in partial denaturation. In an attempt to overcome these difficulties our work provides new avenues to construct reversible functional bio-interfaces. In the following section the applicability of these concepts will be demonstrated for the case of responsive cell-interface studies.

\section{Manipulation of cells via redox-active peptide assemblies on surfaces}

Automated peptide synthesis provides an intrinsic opportunity to very quickly fine-tune properties, such as binding strengths of specific residue(s) to supramolecular hosts. For example, Stupp and co-workers used alginate hydrogel surfaces decorated with CD as host substrate for biomolecule functionalized guest molecules such as naphthol and adamantane [20]. Attaching naphthol to cell adhesive peptide Arg-Gly-Asp (RGD) and adamantane to non-cell adhesion supporting peptide Arg-Gly-Glu-Ser (RGES) they constructed as a competitive surface switch [20]. Using a cell adhesive naphthol-RGDS to bind to such $C D$ substrates, fibroblasts were found to be spreading on the resulting surfaces. When adamantane-RGES was introduced, cells would detach as a consequence of the higher binding strength to $\alpha \mathrm{CD}$ of adamantane, when compared to naphthol (Fig. 1) [20].

In a different system, we have demonstrated the supramolecular control of cell adhesion using RGD peptide-ferrocene conjugates, which were immobilized via host-guest chemistry onto CB[7] SAMs (Fig. 8) [21]. While we know from detailed surface characterization studies, that our $\mathrm{CB}$ [7] SAMs do not reach full coverage of the substrate [18], special attention was required to block the remainder of the surface with cell-repellent molecules (Fig. 8) [21]. When a short thiolated ethylene
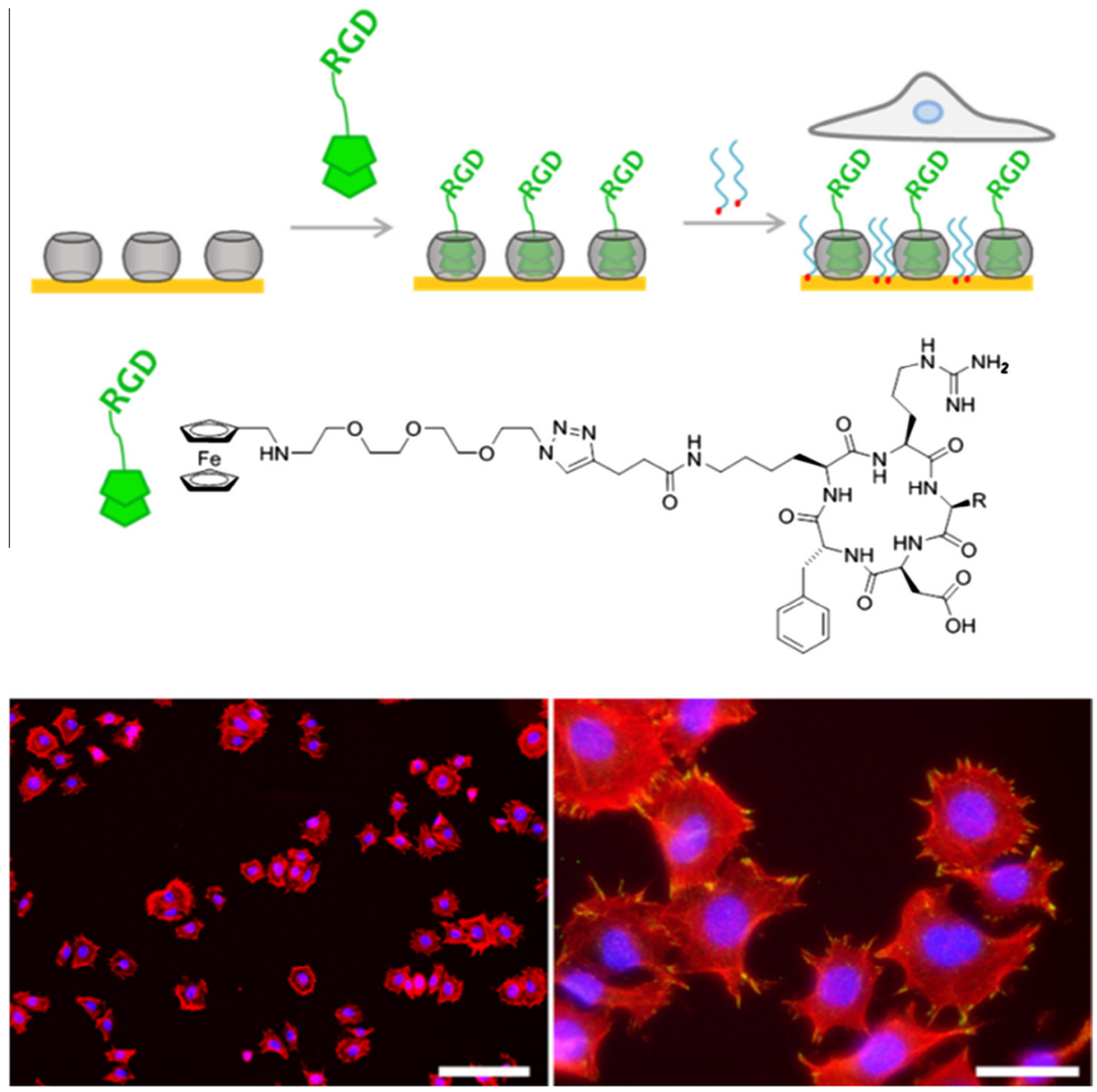

Fig. 8. Coating steps of gold surfaces with $\mathrm{CB}[7]$, ferrocene-modified RGD peptide and blocking agent $\mathrm{EG}_{6} \mathrm{SH}$. Fluorescent micrographs of cells adhered to the indicated surfaces. Scale bar $200 \mu \mathrm{m}$ (left) and $50 \mu \mathrm{m}$ (right). Actin, nuclei and paxillin are stained red, blue and green, respectively. (For interpretation of the references to colour in this figure legend, the reader is referred to the web version of this article.) 


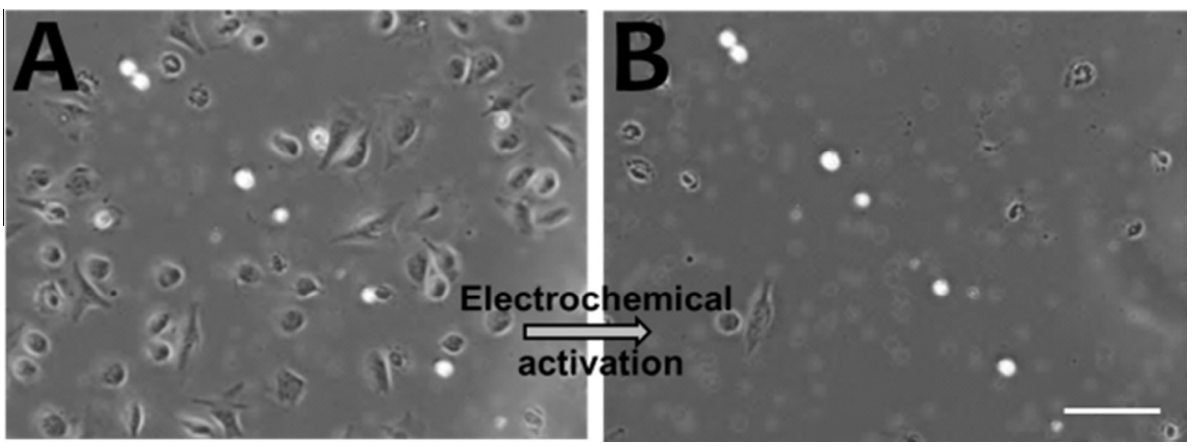

Fig. 9. Bright field images of cells (A) before and (B) after electrochemical detachment at $-0.5 \mathrm{~V}$ ( $\mathrm{vs}$. $\mathrm{Ag} / \mathrm{AgCl}$ ) for $200 \mathrm{~s}$ on supramolecular surfaces made of ternary complexes between surface-bound methyl viologen, $\mathrm{CB}[8]$ and WGGRGDS. The white dots on the substrate serve as markers to indicate the same observation area. The scale bar is $100 \mu \mathrm{m}$. Adopted from Ref. [22].

glycol molecule ( $\left.\mathrm{EG}_{6} \mathrm{SH}\right)$ was used for blocking the surface after binding of ferrocenylamine-peptides to $\mathrm{CB}$ [7] SAMs, specific adhesion and spreading of human umbilical vein endothelial cells was observed [21]. A wound assay on surfaces with CB[7] and ferrocene functionalized RGD showed full recovery of the cell monolayer within $8 \mathrm{~h}$, indicating effective direction of cell growth by the supramolecularly anchored peptide epitopes [21].

As a next step, we employed a Trp-Gly-Gly-Arg-Gly-Glu-Ser (WGGRGDS) peptide to form a ternary 1:1:1 inclusion complex of tryptophan with methyl viologen inside $\mathrm{CB}[8]$. We first immobilized methyl viologen on a substrate. The complex formation lead to the formation of a cell-repellent monolayer, which we verified by attenuated total reflectance infrared spectroscopy (ATR-IR) [22]. In-situ chronoamperometry experiments in our SPR set-up were carried out by applying a potential step to $-0.5 \mathrm{~V}$ (vs. Ag/AgCl), which reduces methyl viologen resulting in the dissociation of the ternary complex [22]. The release of the RGD peptides produced a negative shift of the one-electron reduction potential of methyl viologen [22]. Cells efficiently attached and spread on surfaces that were modified with the ternary RGD complex [22]. When the ternary complex dissociation potential was applied to the substrates over $90 \%$ of the original adherent cells were removed from the surface and the remaining cells displayed a round morphology, indicative of poor adhesion to the surface (Fig. 9) [22]. In addition, spatial and temporal control of the selective complexation of RGD peptides onto the electrodes was demonstrated by modifying parallel lines of gold electrodes with this bioactive ternary complex, while the bare glass substrate in between the electrodes was modified with cell-repellent molecules [22]. Staining of fixed cells with phalloidin showed that their actin filaments were perfectly co-localized with the RGD-modified electrodes [21]. We have shown successfully that we can individually detach specific cells, by applying a suitable voltage pulse to the appropriate electrode [22]. The successful implementation of tools to control surface characteristics with spatial and temporal resolution is of utmost importance to study details of biological processes at the sub-cellular level. The application of supramolecular interactions with biomolecules and biomaterials offers a multitude of possibilities to modulate release kinetics, thermodynamic stabilities and variations in ligand density and ligand mixtures - all compatible with fragile biomolecules, or even cells requiring physiological conditions.

\section{Conclusions and outlook}

Living entities predominantly exist in, interact with and modify a constantly changing environment. This led to the requirement for bioactive ligands to be presented in a highly dynamic manner to mimic the natural environment of eukaryotic and prokaryotic cells. Consequently, we have developed strategies to not only introduce such dynamic properties but also add functional responsiveness, which is the ability of a system to change some properties in response to an external stimulus. Electrochemical stimuli-responsive systems enable us to first achieve and later reverse peptide and protein display. Furthermore, using these concepts, we have demonstrated the ability to change the cellular response, stimulated by an electrochemically caused change in the surface anchoring of cell adhesive peptides. While traditional surface anchoring strategies allow for spatial control of bioactive ligands, dynamics and responsiveness allow for temporal control of these ligands. Very recently the supramolecular stimuli-responsive surface toolbox has been extended with the possibility to optically change cell adhesion employing light-sensitive azobenzene guests [12].

Taking full advantage of the specific anchoring of bioactive ligands using supramolecular host-guest chemistry, specific interaction with cells and subsequent, (locally-)triggered ligand release using redox- and electrochemistry or photon guiding enables the study of the intrinsically responsive processes of cell growth, cell migration and intercellular interactions.

\section{Acknowledgements}

The authors acknowledge the European Research Council for Starters Grant Sumoman (259183) and project P4.02 Superdices of the research program of the BioMedical Materials institute, co-funded by the Dutch Ministry of Economic Affairs, 
Agriculture and Innovation. We are grateful for the fruitful collaboration with Prof. Luc Brunsveld and his co-workers from the Eindhoven University of Technology and discussions with current and previous members of the department of Molecular Nanofabrication of the University of Twente.

\section{References}

[1] J. Brinkmann, E. Cavatorta, S. Sankaran, B. Schmidt, J. van Weerd, P. Jonkheijm, About supramolecular systems for dynamically probing cells, Chem. Soc. Rev. 43 (2014) 4449-4469.

[2] D. Weinrich, P. Jonkheijm, C.M. Niemeyer, H. Waldmann, Applications of protein biochips in biomedical and biotechnological research, Angew. Chem. Int. Ed. 10 (2009) 7744-7751.

[3] J. Cabanas-Danés, J. Huskens, P. Jonkheijm, Chemical strategies for the presentation and delivery of growth factors, J. Mater. Chem. B 2 (2014) $2381-$ 2394.

[4] A. González-Campo, B. Eker, H.J.G.E. Gardeniers, J. Huskens, P. Jonkheijm, A supramolecular approach to enzyme immobilization in micro-channels, Small 8 (2012) 3531-3537.

[5] P. Jonkheijm, D. Weinrich, H. Schroeder, C.M. Niemeyer, H. Waldmann, Chemical strategies for generating protein biochips, Angew. Chem. Int. Ed. 47 (2008) 9618-9647.

[6] J. Lagona, P. Mukhopadhyay, S. Chakrabarti, L. Isaacs, The cucurbit[n]uril family, Angew. Chem. Int. Ed. 44 (2005) 4844-4870.

[7] E. Masson, X. Ling, R. Joseph, L. Kyeremeh-Mensah, X. Lu, Cucurbituril chemistry: a tale of supramolecular success, RSC Adv. 2 (2012) $1213-1247$.

[8] (a) M.V. Rekharsky, Y. Inoue, Complexation thermodynamics of cyclodextrins, Chem. Rev. 98 (1998) 1875-1917;

(b) T. Auletta, M.R. de Jong, A. Mulder, F.C.J.M. van Veggel, J. Huskens, D.N. Reinhoudt, S. Zou, S. Zapotoczny, H. Schönherr, G.J. Vancso, L. Kuipers, $\beta-$ Cyclodextrin host-guest complexes probed under thermodynamic equilibrium: thermodynamics and AFM force spectroscopy, J. Am. Chem. Soc. 126 (2004) 1577;

(c) A. Gomez-Casado, H.H. Dam, M. Deniz Yilmaz, D. Florea, P. Jonkheijm, J. Huskens, Probing multivalent interactions in a synthetic host-guest complex by dynamic force spectroscopy, J. Am. Chem. Soc. 133 (2011) 10849.

[9] S.J. Barrow, S. Kasera, M.J. Rowland, J. Del Barrio, O.A. Scherman, Cucurbituril-based molecular recognition, Chem. Rev. 115 (2015) 12320.

[10] (a) C. Stoffelen, J. Huskens, Soft supramolecular nanoparticles by noncovalent and host-guest interactions, Small 12 (2016) 96-119;

(b) D.A. Uhlenheuer, K. Petkau, L. Brunsveld, Combining supramolecular chemistry with biology, Chem. Soc. Rev. 39 (2010) $2817-2826$.

[11] (a) J. Voskuhl, S. Sankaran, P. Jonkheijm, Optical control over bioactive ligands at supramolecular surfaces, Chem. Commun. 50 (2014) 15144-15447; (b) S. Sankaran, J. van Weerd, J. Voskuhl, M. Karperien, P. Jonkheijm, Photo-responsive cucurbit[8] uril-mediated adhesion of bacteria on supported lipid bilayers, Small 11 (2015) 6187-6196.

[12] (a) C. Stoffelen, J. Voskuhl, P. Jonkheijm, J. Huskens, Dual stimuli-responsive self-assembled supramolecular nanoparticles, Angew. Chem. Int. Ed. 53 (2014) 3400-3404;

(b) F. Tian, D. Jiao, F. Biedermann, O.A. Scherman, Orthogonal switching of a single supramolecular complex, Nat. Commun. 3 (2012) 1207.

[13] I. Hwang, K. Baek, M. Jung, Y. Kim, K.M. Park, D.-W. Lee, N. Selvapalam, K. Kim, Noncovalent immobilization of proteins on a solid surface by cucurbit[7] uril-ferrochenemethylammonium pair, a potential replacement of biotin-avidin pair, J. Am. Chem. Soc. 129 (2007) 4170-4171.

[14] D.A. Uhlenheuer, D. Wasserberg, C. Haase, H.D. Nguyen, J.H. Schenkel, J. Huskens, B.J. Ravoo, P. Jonkheijm, L. Brunsveld, Directed supramolecular surface assembly of SNAP-tag fusion proteins, Chem. Eur. J. 18 (2012) 6788-6794.

[15] D. Wasserberg, D.A. Uhlenheuer, P. Neirynck, J. Cabanas-Danés, J.H. Schenkel, B.J. Ravoo, Q. An, J. Huskens, L.-G. Milroy, L. Brunsveld, P. Jonkheijm, Immobilization of ferrocene-modified SNAP-fusion proteins, Int. J. Mol. Sci. 14 (2013) 4066-4080.

[16] J. Young, H.D. Nguyen, L. Yang, J. Huskens, P. Jonkheijm, L. Brunsveld, Strong and reversible monovalent supramolecular protein immobilization, ChemBioChem 11 (2010) 180-183.

[17] L. Yang, A. Gómez-Casado, J.F. Young, H.D. Nguyen, J. Cabanas-Danés, J. Huskens, L. Brunsveld, P. Jonkheijm, Reversible and oriented immobilization of ferrocene-modified proteins, J. Am. Chem. Soc. 134 (2012) 19199-19206.

[18] A. Gomezcasado, P. Jonkheijm, J. Huskens, Recognition properties of cucurbit[7]uril self-assembled monolayers studied with force spectroscopy, Langmuir 27 (2011) 11508-11513.

[19] A. González-Campo, M. Brasch, D.A. Uhlenheuer, A. Gómez-Casado, L. Yang, L. Brunsveld, J. Huskens, P. Jonkheijm, Supramolecularly oriented immobilization of proteins using cucurbit[8]uril, Langmuir 28 (2012) 16364-16371.

[20] J. Boekhoven, C.R. Pérez, S. Sur, A. Worthy, S.I. Stupp, Dynamic display of bioactivity through host-guest chemistry, Angew. Chem. Int. Ed. 52 (2013) $12077-12080$.

[21] P. Neirynck, J. Brinkmann, Q. An, D. van der Schaft, L. Gustav Milroy, P. Jonkheijm, L. Brunsveld, Supramolecular control over cell adhesion via ferrocene-cucurbit[7]uril host-guest binding on gold surfaces, Chem. Commun. 49 (2013) 3679-3681.

[22] Q. An, J. Brinkmann, J. Huskens, S. Krabbenborg, J. de Boer, P. Jonkheijm, A supramolecular system for the electrochemically controlled release of cells, Angew. Chem. Int. Ed. 51 (2012) 12233-12237. 